\title{
Throughput maximization for full-duplex two-way relay with finite buffers
}

\author{
Betene Anyugu Francis Lin \\ Department of Electronic Engineering, Shanghai Jiao Tong University, China
}

\begin{tabular}{l}
\hline Article Info \\
\hline Article history: \\
Received Feb 11, 2020 \\
Revised Apr 14, 2020 \\
Accepted Apr 29, 2020
\end{tabular}

\section{Keywords:}

Buffer-aided relaying

Full-duplex

Q-learning

Reinforcement learning

Throughput

\begin{abstract}
Optimal queueing control of multi-hop networks remains a challenging problem, especially in two-way relaying systems, even in the most straightforward scenarios. In this paper, we explore two-way relaying having a full-duplex decode-and-forward relay with two finite buffers. Principally, we propose a novel concept based on the multi-agent reinforcement learning (that maximizes the cumulative network throughput) based on the combination of the buffer states and the lossy links; a decision is generated as to whether it can transmit, receive or even simultaneously receive and transmit information. Towards this objective, chiefly, based on the queue state transition and the lossy links, an analytic Markov decision process is proposed to analyze this scheme, and the throughput and queueing delay are derived. Our numerical results reveal exciting insights. First, artificial intelligence based on reinforcement learning is optimal when the length of the buffer is superior to a certain threshold. Second, we demonstrate that reinforcement learning can boost transmission efficiency and prevent buffer overflow.
\end{abstract}

Copyright (c) 2020 Insitute of Advanced Engineeering and Science. All rights reserved.

\section{Corresponding Author:}

Betene Anyugu Francis Lin,

Department of Electrical and Computer Engineering,

Shanghai Jiao Tong University, Shanghai, China.

Email: francislin@sjtu.edu.cn

\section{INTRODUCTION}

With the significant evolution of computer in term of speed, it becomes easy to implement new algorithms to boost sufficiently its performance, artificial intelligence (AI) has become the keyword which defines the future and everything that it holds. Not only has Artificial Intelligence taken over traditional methods of computing, but it has also changed the way industries perform. Recently, AI algorithms have fascinated researchers and have also been applied successfully to solve problems in engineering, such as visual perception, speech recognition, decision-making, and translation between languages. Our research aims to design a new concept of artificial intelligence based on the full-duplex two-way relaying system with reinforcement learning, such that the achievable data rate/throughput is maximized, and the bottleneck problem of buffer overflow is remedied.

Two-way relaying systems, which was first studied by Shannon in [1], adding with the relay to boost the exchange of information between two nodes has been dramatically analyzed and implemented to overcome the incessant demand of speed. In [2-10], the capacity of the two-way relay channel (TWRC) in half-duplex and full-duplex (FD) has been extensively investigated, and the average throughput and delays of the proposed protocols were evaluated. With the benefit of AI, for cooperative wireless networks, in half-duplex. Reinforcement learning $(\mathrm{RL})$ is used to increase the performance of cooperative wireless networks. In [11], the authors introduced the framework of communication systems and the jamming modes commonly used in communica- 
tion. Then the basic principle of the quality-learning(Q-Learning) algorithm is briefly introduced. However, they didn't analyze the throughput and system delay. In [12], the authors examined the problem of relay node selection in cooperative networks, in which one relay node can be used by multiple source-destination transmission pairs, and all transmission pairs share the same set of relay nodes. However, the throughput was not optimized. In[13], the authors investigate the power control problem in a cooperative network with multiple wireless transmitters, multiple amplify-and-forward relays, and one destination. Nevertheless, the power control problem in a cooperative network for the two-way relay was not investigated. The goal is to maximize the long-term system throughput by fully exploiting multi-user diversity in the network. Furthermore, the delay performance of the network, which is very crucial to evaluate the system network was not investigated. Whereas in $[14,15]$, the authors present a novel deep reinforcement learning-based joint spectrum sensing and power control algorithm for downlink communications in a cognitive small cell. Regrettably, the authors did not investigate the outage probability for the whole system. In[16], the authors propose an iterative optimal power allocation (OPA) method to maximize the derived end-to-end sum rate, which is based on Lagrangian and Newton-Raphson algorithm under total power constraint. The authors used reinforcement learning for two-way relaying systems. However, the system delay was not investigated. However, in [17], the authors investigated two-way practical relay protocol with an enhanced transmission scheduling, which takes a joint consideration of the finite relay buffers, signalling overheads, and lossy links.

In this regard, to improve the performance of the system, full-duplex two-way relaying with buffer is proposed due to its throughput merits. However, the authors in [18] propose a novel adaptive protocol (that maximises the cumulative network throughput) based on the combination of the buffer states, lossy link, and the outage probability; a decision is generated as to whether it can transmit, receive or even simultaneously receive and transmit information. Similarly, they investigated the queue state, the transition matrix, an analytic Markov chain model was proposed to analyse this scheme, and the throughput and queueing delay were derived. From the available literature, we are the first and the only one to have proposed a concept of the full-duplex two-way relay (FD-TWR) with reinforcement learning, such that the achievable data rate/throughput is maximized.

The contribution of this paper can be summarized as follows:

- We propose a reinforcement learning approach for full-duplex two-way relay based on multi-agents. As far as we know, we are the first to introduce the use of reinforcement learning for the full-duplex two-way relay, and we do not only take into consideration the instantaneous qualities of the involved links but also consider the states of the queues at the buffers for the full-duplex two-way relay.

- We present a general framework for obtaining the average throughput and the system delay based on Markov decision process of the queue states at the buffers.

\section{RESEARCH METHOD}

\subsection{Channel models}

In this paper, throughout this paper, we assume that source and destination always transmit data, we investigate the maximum rate of the three-node model-based full-duplex two-way relay(FD-TWR) with finite buffers in the decode-and-forward mode, denoted as $Q_{a}$ and $Q_{b}$. There is no direct link between node A and node B. We consider the FD-TWR with finite buffer-aided where two users named A and B exchange data via the relay $(\mathrm{R})$,which is equipped with two finite buffers, see Figure 1.

On another note, we denote $T$ as the state when the relay transmits data, $R$ as the state when the relay receives data and $T R$ as the state when the relay transmits and receives data at the same time. In parallel, we denote $E$ as the state when the buffers are empty, $F$ as the state when the buffers are full, and $F E$ state of the buffers when the buffers are neither empty nor full. We denote the channel coefficients as $h_{A R}, h_{B R}$, $h_{R R}$ representing the channels from the source $\mathrm{A}$ to the relay $\mathrm{R}$ and from the source $\mathrm{B}$ to the relay $\mathrm{R}$, and the self-interference, respectively. Namely, the relay can transmit and receive data at the same time. Moreover, due to the co-channel transmission and imperfect interference cancellation, we assume that the FD-TWR would undergo more severe self-interference.

Thus, in essence, one of the byproducts of this work is to, unlike in [19], assume that user A and user $\mathrm{B}$ have enough information to send to the relay in some time slots, and do not have enough information in other time slots. Especially, we assume that the state of buffers can also be empty or full in all time slots. Finally, for the theoretical approach, we assume that the full-duplex relay is perfect, that means that $h_{R R}=0$ and $h_{A R}$, $h_{B R}$ are binary dependent variables. 


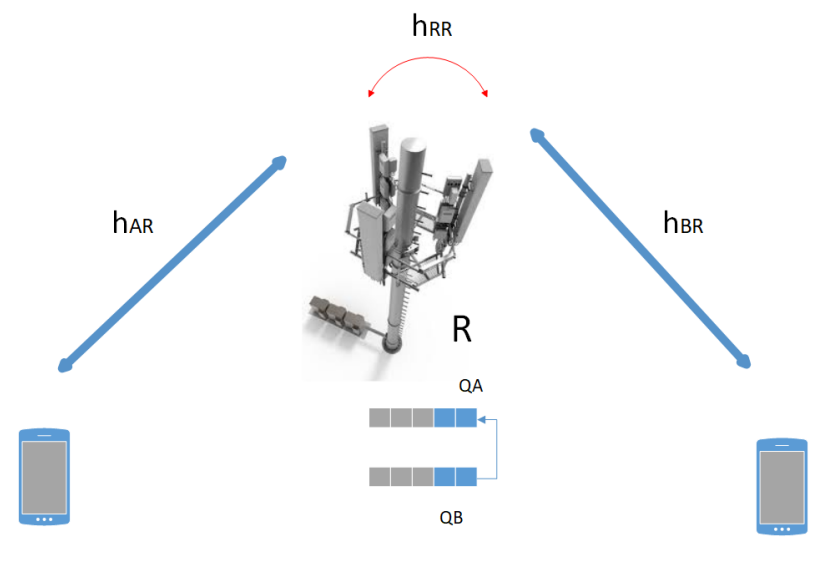

A

B

Figure 1. System model

\subsection{Reinforcement learning for multi-agent}

In this section, unlike [11-15] where unsupervised and supervised learning are used, we implement the system model based on reinforcement learning, as shown in Figure 2.
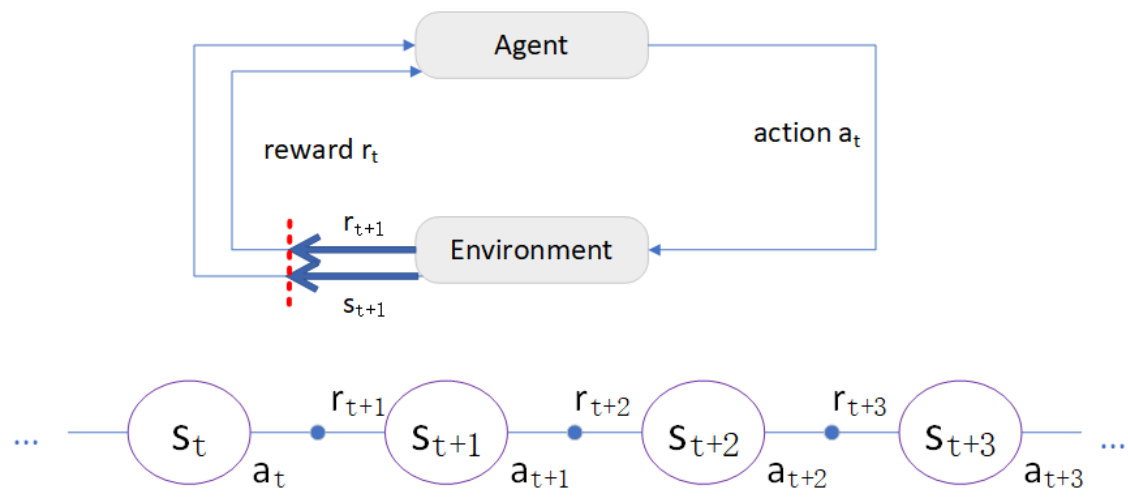

Figure 2. Reinforcement learning cycle

The current state of the environment which the agent observers is defined as $s_{t}$, the action of the agent is defined as $a_{t}$. The reward (or penalty) received by the action $a_{t}$ reflects how perfect the previous action $a_{t-1}$ in the environment $s_{t-1}$. The model of the single-agent RL is a Markov decision process. It is defined as:

$$
f: S \times A \rightarrow[0,1] \rho: S \times A \rightarrow \Re
$$

where $S$ is the finite set of the environment states, $A$ is the finite set of agent actions, $f$ is the state transition probability function, $\rho$ is the reward function. For the dynamic environment, the transition probability function and the reward are: where $s_{t}$ and $s_{t-1}$ are the environment states at $\mathrm{t}$ and $t-1, A_{t}$ is the agent actions at time $\mathrm{t}, f_{t}$ is the state transition probability function, $p_{t}$ is the reward function. The behavior of the agent is described by the policy $\pi$, which specifies how does the agent choose its actions from the given state. The policy may be either static and dynamic. In static case $\pi=S \rightarrow A$, the policy is stationary. In a dynamic case, $\pi_{t}: \mathbb{S} \times \mathbb{A} \rightarrow[0,1]$, the policy is time-varying. The path planning is to find a policy such that the return $\mathrm{R}$ is maximized for the state $S$,

$$
R^{\pi}=E\left\{\sum_{k=0}^{\infty} \gamma_{r k+1}^{k} \mid s_{0}=s, \pi\right\}
$$


where $\gamma \epsilon[0,1)$ is the discount factor. This form is taken over the probabilistic state transition under the policy $\pi$. $R$ also represents the reward accumulated by the agent. The task of the agent is, therefore, to maximize its long-term performance. It is obtained by computing the optimal state action-value function, called Q-function. The Q-function is presented as $\mathbb{Q}^{h}: \mathbb{S} \times \mathbb{A} \rightarrow \mathbb{R}$

$$
Q^{\pi}(s, a)=E\left\{\sum_{k=0}^{\infty} \gamma_{r k+1}^{k} \mid s_{0}=s, a_{0}=a, \pi\right\}
$$

The optimal Q-function is defined as

$$
Q^{\times}(s, a)=\max _{\pi} Q^{\pi}(s, a)
$$

$Q^{*}(s, a)$ can be written as

$$
\begin{aligned}
V^{*}(s) & :=\max _{a \in \mathcal{A}(s)} Q^{*}(s, a) \\
& =\max _{a} \mathbb{E}_{\pi^{*}}\left[\sum_{k=0}^{\infty} \gamma^{k} r_{t+k+1} \mid s_{t}=s, a_{t}=a\right] \\
& =\max _{a} \mathbb{E}_{\pi^{*}}\left[r_{t+1}+\gamma \sum_{k=0}^{\infty} \gamma^{k} r_{t+k+2} \mid s_{t}=s, a_{t}=a\right] \\
& =\max _{a} \mathbb{E}_{\pi^{*}}\left[r_{t+1}+\gamma V^{*}\left(s_{t+1}\right) \mid s_{t}=s, a_{t}=a\right] \\
& =\max _{a} \sum_{s^{\prime} \in S} P\left(s^{\prime} \mid s, a\right)\left[R\left(s, a, s^{\prime}\right)+\gamma V^{*}\left(s^{\prime}\right)\right]
\end{aligned}
$$

In the multi-agent case, the state transitions are the result of joint actions $a_{k}$ within all agents, $a_{k}$

$$
\begin{aligned}
& \mathbb{P}: \mathbb{S} \times \mathbb{A} \times \mathbb{S} \rightarrow \mathbb{R} \\
& f: S \times A \times S \rightarrow\left[\begin{array}{ll}
0 & 1
\end{array}\right] \\
& A=A_{1} A_{2} \ldots \times A^{n}
\end{aligned}
$$

The joint policy is defined as $\pi$. The return of the multi-agent depends on the joint policy is

$$
\begin{gathered}
a_{k}=\left[a_{1, K}^{T}, \ldots, a_{n, K}^{T}\right]^{T} \\
a_{k} \in A, a_{i, k} \epsilon A_{i} \\
R_{\pi}^{i}(x)=E\left\{\sum_{k=0}^{\infty} \gamma_{r k+1}^{k} \mid s_{0}=s, \pi\right\}
\end{gathered}
$$

The Q-function of each agent depends on the joint action and the join policy $Q_{i}^{\pi}: S \times A \rightarrow \mathbb{R}$

$$
Q_{\pi}^{i}(s, a)=E\left\{\sum_{k=0}^{\infty} \gamma_{r k+1}^{k} \mid s_{0}=s, a_{0}=a, \pi\right\}
$$

The transition diagram can be represented as, as shown in Figure 2. 


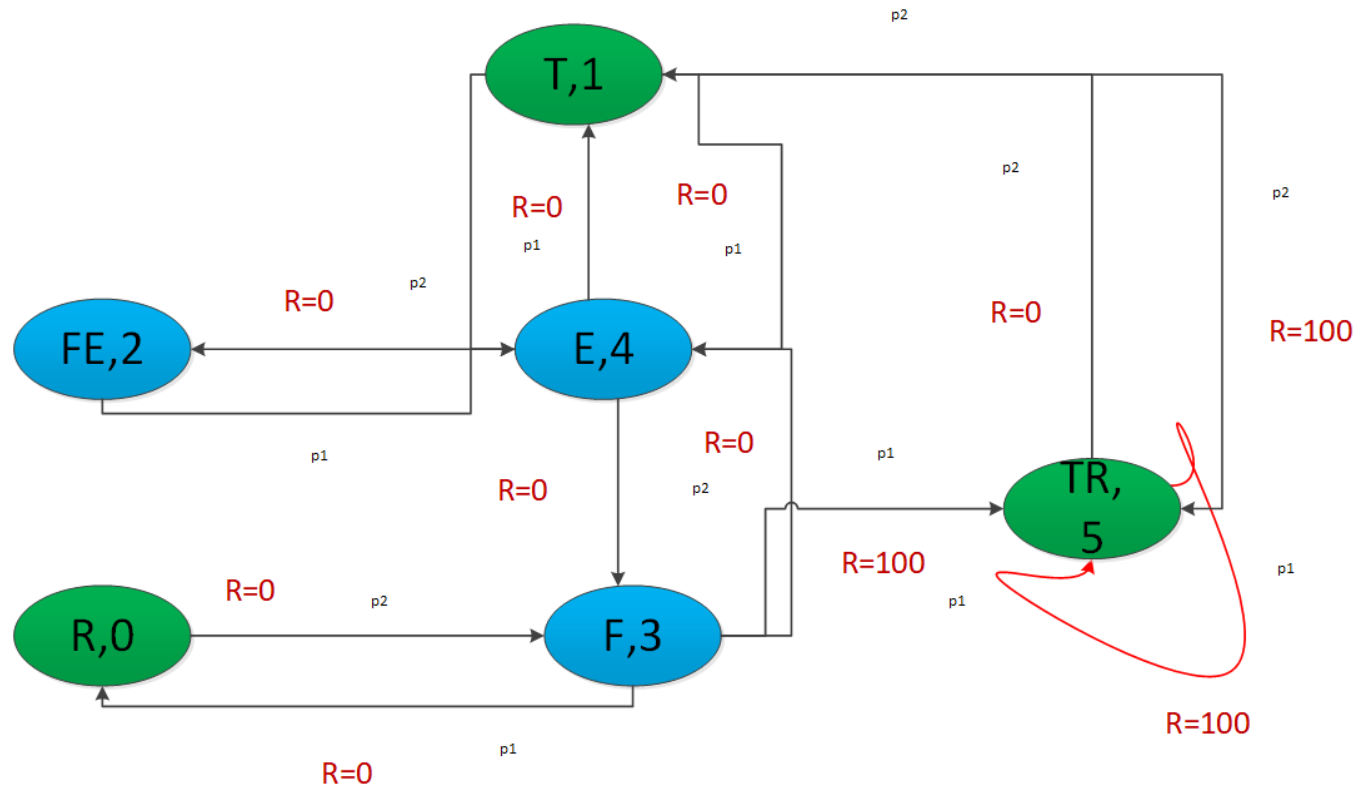

Figure 3. Transition diagram

\subsection{Throughput-delay analysis}

In this section, we present a general framework for the throughput maximization. For simplicity, the Markov process decision is reducible since there are isolated states in the Markov process decision. We can represent all this mechanics on a reward table. On this table, the rows represent the rooms, and the columns represent the actions. The values on this matrix represent the rewards, the value (-1) indicates that some specific actions are not available. We assume that the probability of moving for one state to another can be represented as $p 1$ and $p 2$. Therefore, for simplicity, we assume that $p 1=p 2$. Consequently, we can represent all this mechanics on a reward table $R$

$$
R=\left[\begin{array}{cccccc}
-1 & -1 & -1 & -1 & 0 & -1 \\
-1 & -1 & -1 & 0 & -1 & 100 \\
-1 & -1 & -1 & 0 & -1 & -1 \\
-1 & 0 & 0 & -1 & 0 & -1 \\
0 & -1 & -1 & 0 & -1 & 100 \\
-1 & 0 & -1 & -1 & 0 & 100
\end{array}\right]
$$

We have 3 possible actions: 1,4 or 5 , with their rewards $0,0,100$. It is merely all positive values from row "5", and we are just interested in the one with the most significant value. Then, we select the biggest $Q$ value with those possible actions by selecting $\mathrm{Q}(5,1), Q(5,4), Q(5,5)$.

$$
Q=\left[\begin{array}{cccccc}
0 & 0 & 0 & 0 & 0 & 0 \\
0 & 0 & 0 & 0 & 0 & 100 p 1 \\
0 & 0 & 0 & 0 & 0 & 0 \\
0 & 0 & 0 & 0 & 0 & 0 \\
0 & 0 & 0 & 0 & 0 & 0 \\
0 & 0 & 0 & 0 & 0 & 0
\end{array}\right]
$$

By selecting the action "1" as our next state will have now the following possible actions

$$
Q=\left[\begin{array}{cccccc}
0 & 0 & 0 & 0 & 0 & 0 \\
0 & 0 & 0 & 0 & 0 & 100 p 1 \\
0 & 0 & 0 & 0 & 0 & 0 \\
0 & 80 & 0 & 0 & 0 & 0 \\
0 & 0 & 0 & 0 & 0 & 0 \\
0 & 0 & 0 & 0 & 0 & 0
\end{array}\right]
$$




$$
\begin{gathered}
Q=\left[\begin{array}{cccccc}
0 & 0 & 0 & 0 & 0 & 400 p 1 \\
0 & 0 & 0 & 0 & 320 p 1 & 100 p 1 \\
0 & 0 & 0 & 0 & 320 p 1 & 0 \\
0 & 400 p 1 & 256 p 1 & 0 & 0 & 500 p 1 \\
320 p 1 & 0 & 0 & 0 & 320 p 1 & 500 p 1 \\
0 & 400 p 1 & 0 & 0 & 0 & 0
\end{array}\right] \\
Q=(3,1)+0.8 \cdot \max ([Q(1,3), Q(1,5))] p_{1}=100+0.80
\end{gathered}
$$

After a lot of episodes(100000) our Q matrix can be considered to have convergence, in this case Q will be like this:

$$
Q=\left[\begin{array}{cccccc}
0 & 0 & 0 & 0 & 80 p 1 & 0 \\
0 & 0 & 0 & 64 p 1 & 0 & 100 p 1 \\
0 & 0 & 0 & 64 p 1 & 0 & 0 \\
0 & 80 p 1 & 51 p 1 & 0 & 80 p 1 & 0 \\
64 & 0 & 0 & 64 p 1 & 0 & 100 p 1 \\
0 & 80 p 1 & 0 & 0 & 80 p 1 & 100 p 1
\end{array}\right]
$$

Then, we define the throughput $\phi$ as the amount of successfully delivered data of both A and B per second, which can be given by

$$
\begin{aligned}
\phi & =\sum_{1}^{Q_{a}} \sum_{1}^{Q_{b}} Q^{*}(s, a) T_{Q b}^{Q a} \\
& =\sum_{1}^{Q_{a}} \sum_{1}^{Q_{b}} \max _{\pi} Q^{\pi}(s, a) T_{Q b}^{Q a} \\
& =\sum_{1}^{Q_{a}} \sum_{1}^{Q_{b}} \max _{a} \sum_{s^{\prime} \in S} P\left(s^{\prime} \mid s, a\right)\left[R\left(s, a, s^{\prime}\right)+\gamma V^{*}\left(s^{\prime}\right)\right](s, a) T_{Q b}^{Q a}
\end{aligned}
$$

where $\mathrm{T}$ is the fixed transmission rate of the nodes. After that, we denote $Q$ as the average length of buffers, then we have

$$
\begin{aligned}
Q & =\sum_{a=1}^{a} \sum_{b=1}^{b} n_{a, b} \pi_{a, b}=\sum_{k=1}^{n} k p^{k} \\
& =\frac{p\left(n p^{(n+1)}-(n+1) p^{n}+1\right)}{(p-1)^{2}} .
\end{aligned}
$$

The last equation in (16) can be proved by induction as follows.

When $n=1$, we have

$$
p=\frac{p\left(p^{1+1}-(1+1) p+1\right)}{(p-1)^{2}}=\frac{p\left(p^{2}-2 p+1\right)}{(p-1)^{2}} .
$$

Assume it holds for $n, n \geq 2$, then for $n+1$, we have 


$$
\begin{aligned}
Q & =(n+1) P^{n+1}+\frac{p\left(n p^{n+1}-(n+1) p^{n}+1\right)}{(p-1)^{2}} \\
& =\frac{(n+1) p^{n+1}(p-1)^{2}+p\left(n p^{n+1}-(n+1) p^{n}+1\right)}{(p-1)^{2}} \\
& =\frac{p\left((n+1) p^{n+2}-(n+1+1) p^{n+1}+1\right)}{(p+1)^{2}} \\
& =\sum_{k=1}^{n+1} k p^{k} .
\end{aligned}
$$

Then (16) has been proved.

Finally, the average packet delay $T$ can be properly defined according to the Little's Law in [25], i.e.,

$$
T=\frac{Q}{\phi}
$$

\section{RESULTS AND DISCUSSION}

In this section, we evaluate the performance of the FD-TWR aided buffer in terms of the throughput and the system delay according to the formulas derived in the above section. For the simulations, the size of the buffers $(N)$ is finite, and we adopt $N=40$ in all simulations. $T_{Q b}^{Q a}$ is a variable rate, $T=5$ is a fixed rate, and $p 1=p 2=1$

Figure 4 compares the system delay between FD-TWR aided buffer with Q-learning and FD-TWR aided buffer without Q-learning versus the size of the buffers. It reveals that when the size of the buffers increases, the system delay is reduced, and the average packet delay of the full-duplex with Q-learning relay is smaller than the one without Q-learning. That means that with Q-learning after a certain threshold value 15, the size of the buffer doesn't affect the performance of the system; this is due to the reinforcement learning algorithm.

Figure 5 compares the throughput between FD-TWR aided buffer with Q-learning and the throughput of FD-TWR aided buffer without Q-learning versus the size of the buffers. versus the number of the buffer. The results show that when the buffer size is 5 , the throughput is constant for both curves, this is due to the optimization of the full-duplex relay when it can receive and send at the same time. Moreover, we observe that there is a correlation between the two curves. Both curves are steady, and the throughput of FD-TWR aided buffer with Q-learning overtakes the one without a Q-learning.

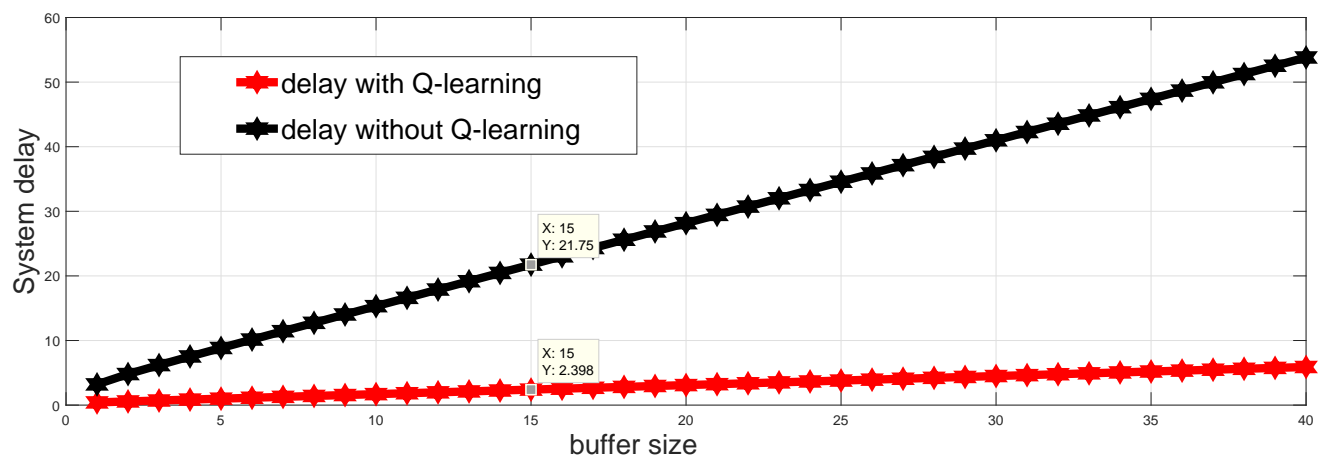

Figure 4. System delay versus buffer size 


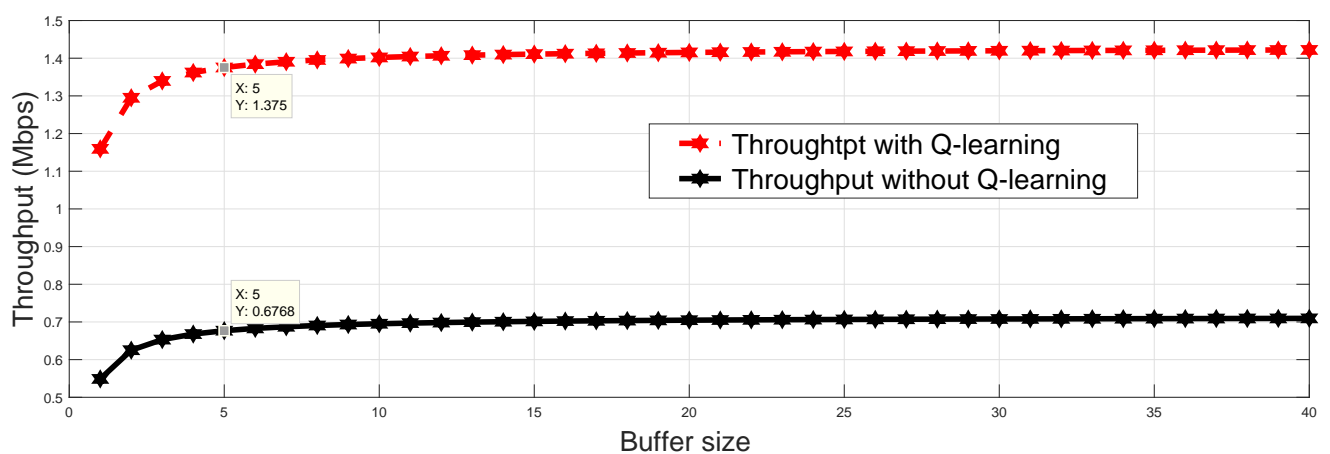

Figure 5. Throughput with Q-learning versus versus buffer size

\section{CONCLUSION}

In this paper, we studied the throughput and the system delay in FD-TWR networks using a reinforcement learning scheme. Specifically, we considered a buffer-aided-relay which has a finite length buffer. Based on the Markov decision process, we characterized a reinforcement learning approach that can maximize the aggregate network throughput by taking into consideration the constraint of the buffer state. Moreover, we compared the performance of the proposed transmission policy to the FD-TWR with the reinforcement learning approach and the one without the reinforcement learning scheme, especially when our full-duplex relay has finite buffers. The numerical results showed that buffering relay techniques improve the capacity of relay networks in slow fading environments, and the reinforcement learning scheme significantly improves the throughput of the system by queuing or dequeuing the buffers rapidly according to the buffer states and lossy link. Furthermore, the reinforcement learning scheme does not need too much buffer size to maximize average throughput and the system delay. It also remedies the bottleneck problem of buffer overflow.

\section{ACKNOWLEDGMENTS} Council).

This research was supported by Project 1236130208, supported by the CSC (China Scholarship

\section{REFERENCES}

[1] C. E. Shannon, et al., "Two-way communication channels," Proc. 4th Berkeley Symp. Math. Stat. Prob, vol. 1, pp. 611-644, 1961.

[2] G. Kramer, et al., "Cooperative communications," Foundations and Trends R? in Networking, vol. 1, no. 3-4, pp. 271-425, 2007.

[3] P. Popovski and H. Yomo, "Physical network coding in two-way wireless relay channels," IEEE International Conference on Communications, pp. 707-712, 2007.

[4] L. Ong, "The half-duplex gaussian two-way relay channel with direct links," IEEE International Symposium on Information Theory, pp. 1891-1895, 2015.

[5] L. Ding, M. Tao, F. Yang, and W. Zhang, "Joint scheduling and relay selection in one-and two-way relay networks with buffering," IEEE International Conference on Communications, pp. 1-5, 2009.

[6] H. Liu, P. Popovski, E. De Carvalho, and Y. Zhao, "Sum-rate optimization in a two-way relay network with buffering," IEEE Communications Letters, vol. 17, no. 1, pp. 95-98, 2013.

[7] Y. Gu and S. Aissa, "Interference aided energy harvesting in decode-and-forward relaying systems," IEEE international conference on communications, pp. 5378-5382, 2014.

[8] N. Zlatanov, D. Hranilovic, and J. S. Evans, "Buffer-aided relaying improves throughput of fullduplex relay networks with fixed-rate transmissions," IEEE Communications Letters, vol. 20, no. 12, pp. 2446-2449, 2016.

[9] K. T. Phan and T. Le-Ngoc, "Power allocation for buffer-aided full-duplex relaying with imperfect selfinterference cancelation and statistical delay constraint," IEEE Access, vol. 4, pp. 3961-3974, 2016. 
[10] M. M. Razlighi and N. Zlatanov, "Buffer-aided relaying for the two-hop full-duplex relay channel with self-interference," IEEE Transactions on Wireless Communications, vol. 17, no. 1, pp. 477-491, 2018.

[11] Z. Zhang, Q. Wu, B. Zhang, and J. Peng, 'Intelligent anti-jamming relay communication system based on reinforcement learning," 2nd International Conference on Communication Engineering and Technology, pp. 52-56, 2019.

[12] Z. Chen, T. Lin, and C. Wu, "Decentralized learning-based relay assignment for cooperative communications," IEEE Transactions on Vehicular Technology, vol. 65, no. 2, pp. 813-826, 2015.

[13] F. Shams, G. Bacci, and M. Luise, "Energy efficient power control for multiple-relay cooperative networks using q-learning," IEEE Transactions on Wireless Communications, vol. 14, no. 3, pp. 1567-1580, 2014.

[14] X. Meng, H. Inaltekin, and B. Krongold, "Deep reinforcement learning-based power control in full-duplex cognitive radio networks," IEEE Global Communications Conference (GLOBECOM), pp. 1-7, 2018.

[15] X. Qiu, T. Jiang, and N. Wang, "Safeguarding multiuser communication using full-duplex jamming and q-learning algorithm," IET Communications, vol. 12, no. 15, pp. 1805-1811, 2018.

[16] K. Chang and Y. Choi, "Performance evaluation of in-band full-duplex system using one-time-slot twoway relay," IEEE Systems Journal, 2019.

[17] S. Shi, S. Li, and J. Tian, "Markov modeling for practical two-way relay with finite relay buffer," IEEE Communications Letters, vol. 20, no. 4, pp. 768-771, 2016.

[18] B. A. F. Lin, X. Ye, and S. Hao, "Adaptive protocol for full-duplex two-way systems with the buffer-aided relaying," IET Communications, vol. 13, no. 1, pp. 54-58, 2018.

[19] V. Jamali, N. Zlatanov, and R. Schober, "Bidirectional buffer-aided relay networks with fixed rate transmissionpart ii: Delay-constrained case," IEEE Transactions on Wireless Communications, vol. 14, no. 3, pp. 1339-1355, 2015.

[20] A. Shafeeq and K. Hareesha, "Dynamic clustering of data with modified k-means algorithm," Proceedings of the 2012 conference on information and computer networks, pp. 221-225, 2012.

[21] N. Kamari, I. Musirin, Z. Hamid, and A. A. Ibrahim, "Optimal tuning of svc-pi controller using whale optimization algorithm for angle stability improvement," Indonesian Journal of Electrical Engineering and Computer Science, vol. 12, no. 2, pp. 612-619, 2018.

[22] M. M. Saufi, M. A. Zamanhuri, N. Mohammad, and Z. Ibrahim, "Deep learning for roman handwritten character recognition," International Journal of Electrical Engineering and Computer Science, vol. 12, no. 2, pp. 455-460, 2018.

[23] A. H. Basori, A. Tenriawaru, and A. B. F. Mansur, "Intelligent avatar on e-learning using facial expression and haptic," TELKOMNIKA Telecommunication, Computing, Electronics and Control, vol. 9, no. 1, 2011.

[24] R. Luo, W. Liao, and Y. Pi, "Discriminative supervised neighborhood preserving embedding feature extraction for hyperspectral-image classification," TELKOMNIKA Telecommunication, Computing, Electronics and Control, vol. 10, no. 5, pp. 1051-1056, 2012.

[25] J. D. Little, "Or forumlittle's law as viewed on its 50th anniversary," Operations research, vol. 59, no. 3, pp. 536-549, 2011.

\section{BIOGRAPHIES OF AUTHORS}

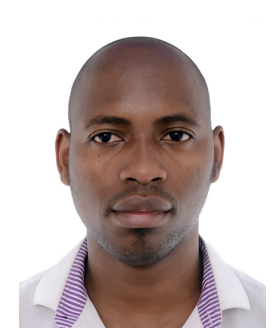

Francis BETENE is a professional research assistant at REMI-Ultra-electronics with a Master of science from Hohai university China (2013). He obtained a PhD Degree in wireless technologies from Shanghai Jiao-Tong university (China) in 2019. His researches are in fields of electronics, digital systems, wireless, signal processing, and artificial intelligence. Recently, system's application on reinforcement learning has been tackled. He is affiliated with IEEE as reviewer member. In IJECE, IAES journals, and other scientific publications, he has served as invited reviewer. 